\section{IL GRAFFIO La conta dei morti}

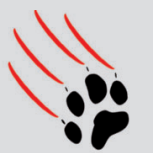

Tra i fattori determinanti della salute (individuale, familiare e sociale) il livello di istruzione ("education" dicono gli anglosassoni, dando forse alla parola un significato più ampio della semplice scolarizzazione) gioca un ruolo altissimo. Forse il più importante: perché l'istruzione, oltre che assicurare la messa in atto di meccanismi protettivi verso le avversità ambientali, materiali ed emozionali, favorisce di per sé l'avvio di circoli virtuosi, come ad esempio quello del miglioramento della posizione socioeconomica. In particolare (forse lo sapevamo gia o forse lo avevamo solo distrattamente orecchiato...), anche la mortalità infantile sembra fortemente correlata al livello educativo del contesto familiare in cui cresce il bambino e in particolare al grado di istruzione dei genitori. Questa evidenza, che magari può sembrare scontata ma delle cui ricadute non sembra proprio ci sia, forse nemmeno tra di noi, un'adeguata consapevolezza (tantomeno sul piano operativo) ci viene ora violentamente rappresentata dai risultati di uno studio a dir poco colossale. Si tratta di una revisione di 186 studi relativi al rapporto tra grado di istruzione parentale e mortalità infantile sotto 5 anni (selezionati, per adeguatezza metodologica, tra 5339 studi in quindici lingue e relativi a 92 Paesi), integrata dall'analisi dei dati demograficie di salute di tremilionicentododicimilaquattrocentosettantaquattro nati (Balaj $M$, et al. Parental education and inequalities in child mortality: a global systematic review and meta-analysis. Lancet 2021;398(10300): 608-20. doi: 10.1016/S0140-6736(21)00534-1). Indipendentemente dai fattori potenzialmente confondenti come il livello socioeconomico il sesso del bambino, il livello di istruzione dei genitori risulta avere, $d a$ solo, un potente effetto protettivo sulla mortalità infantile (... mortalità! vi prego di fare attenzione, parliamo di vita o morte, senza vie di mezzo!): la riduzione, rispetto ai bambini con genitori non scolarizzati, è del $31 \%$ per i bambini nati da madri che hanno completato il ciclo scolastico di 12 anni e del $17,3 \%$ se ad aver raggiunto questo grado di scolarizzazione è almeno il padre. L'effetto doselrisposta è lineare: ogni anno di scolarizzazione della madre vale il $3 \%$ di mortalità in meno, ogni anno di scolarizzazione del padre l'1,5\%. Non c'è molto da aggiungere, ci sarebbe (in questo caso si) molto da urlare. E anche da fare (pediatri o non pediatri che si sia o ci si dica). In fin dei conti, l'istruzione (con la crescita socioculturale e la salute che attraverso di questa viene garantita a tutti nello stesso modo) rappresenta proprio uno degli obiettivi assoluti, laici (e quindi, sì, senza dubbio esportabili) della democrazia. Un fondamento che ne sottende un altro, altrettanto assoluto, altrettanto laico: quello dell'uguaglianza dei diritti tra tutti, senza distinzioni e discriminazioni, prima di tutto senza distinzioni e discriminazioni tra uomini e donne. La mortificazione di genere, la cui violenza ci è ora riproposta dal veto scolastico alle bambine imposto dai Talebani in Afghanistan (ma di fatto, e in diversi modi, agito anche in tante altre parti del mondo) nega il senso della vita e la vita stessa a milioni di bambine e di donne. Ma, al contempo, proprio per questo, annichilisce le prospettive di vita e sviluppo di tutte le diverse società: anche di quelle che, come la nostra, potrebbero ancora incorrere nel disumano (e catastrofico) errore di minimizzare, di rimandare ogni azione concreta, limitandosi ad agire il previlegio di poter contare $i$ morti delle altre... 